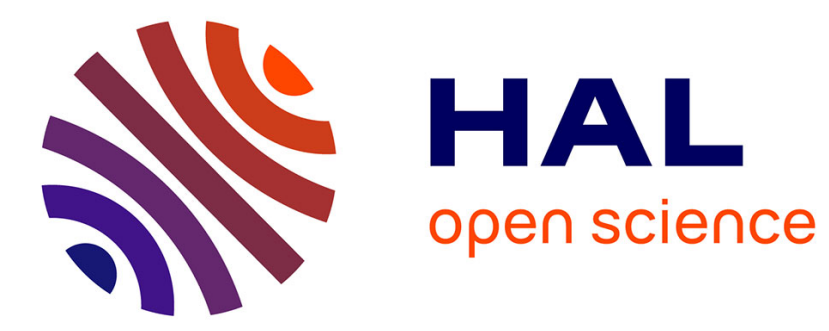

\title{
Swimming force characterizations of multistaged bi-helical microswimmer and 3D vortex trap manipulation
}

\author{
Alisier Paris, Dominique Decanini, Gilgueng Hwang
}

\section{To cite this version:}

Alisier Paris, Dominique Decanini, Gilgueng Hwang. Swimming force characterizations of multistaged bi-helical microswimmer and 3D vortex trap manipulation. Microelectronic Engineering, 2020, 235, pp.111466. 10.1016/j.mee.2020.111466 . hal-03020648

\section{HAL Id: hal-03020648 https://hal.science/hal-03020648}

Submitted on 24 Nov 2020

HAL is a multi-disciplinary open access archive for the deposit and dissemination of scientific research documents, whether they are published or not. The documents may come from teaching and research institutions in France or abroad, or from public or private research centers.
L'archive ouverte pluridisciplinaire HAL, est destinée au dépôt et à la diffusion de documents scientifiques de niveau recherche, publiés ou non, émanant des établissements d'enseignement et de recherche français ou étrangers, des laboratoires publics ou privés. 


\section{Swimming force characterizations of multistaged bi-helical microswimmer and 3D vortex trap manipulation}

Alisier Paris ${ }^{1}$, Dominique Decanini ${ }^{1}$, Gilgueng Hwang ${ }^{1,2,3 *}$

${ }^{1}$ C2N - CNRS, University Paris-Sud, University Paris-Saclay, Palaiseau, France

${ }^{2}$ LIMMS-CNRS, Institute of Industrial Science, The University of Tokyo, Japan

${ }^{3}$ Dept. of Electrical Engineering and Information Systems, The University of Tokyo, Japan

${ }^{*}$ Corresponding author. E-mail: gilgueng.hwang@c2n.upsaclay.fr Phone: (33)-6-7575-4072

GRAPHICAL ABSTRACT:

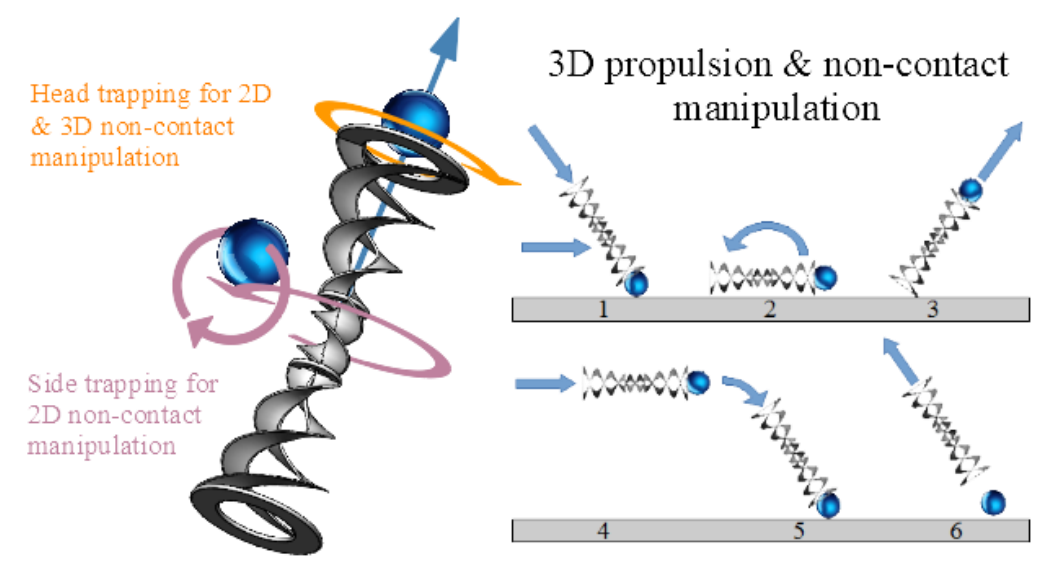




\begin{abstract}
Vortex trap manipulation of microscopic objects in three-dimensions by helical microswimmers has a great potential towards non-contact biological cell manipulation or microassembly. However, in the current state-of-the-art, it has been limited in 2D manipulation due to the conflicting characteristics of optimizing the trapping force and propulsion force. In this paper, we propose a new design of the helical microswimmers enabling purely non-contact, selective and 3D vortex trap micromanipulation. The proposed helical microswimmers are fabricated by 3D nanoprinting technology based on twophoton laser absorption. The vertically standing helical mirostructures on top of the supporting micropillars allows uniform coating of ferromagnetic metal layer with minimum shadow area during metallization by sputtering. Furthermore this reduces the risk of damaging or losing materials during micromanipulation process for releasing them after fabrication which allows propulsion force characterizations and optimization. We characterized to reveal their propulsion force and this proved the propulsion force was recovered back to even higher than the single helical microswimmers. We consider that the proposed helical microswimmers with 3D manipulation could have a great impact to non-contact biological cell manipulation.
\end{abstract}

\title{
KEYWORDS
}

Helical microswimmer, multistaged bi-helix, two-photon 3D lithography, magnetic actuation, noncontact 3D manipulation, hydrodynamic vortex trap 


\section{MAIN TEXT}

\section{Introduction}

The manipulation of microorganisms (bacteria, yeasts, etc.) or individual cells has always been a challenge and a limitation for biologists and physicians. Yet it has been shown that mechanical interactions play an important role in cell life [1]-[4]. Thus, having an effective manipulation tool should open up many opportunities that go far beyond simple study and organization. For example: the measurement of the stiffness of a cell wall can predict the risk of cancerous transmission [5] and the application of mechanical force to a tumor can control its evolution [6], the modulation of the force applied on a cell makes it possible to play on its mitosis [7], [8] and thus on the mechanisms of genetic reproduction, or, more simply, the manipulation of gametes can greatly facilitate in-vitro reproduction. All these examples require having a very precise manipulation tools that can act in three dimensions at the microscopic scale in a highly controlled environment. Also, over time, different techniques have emerged to allow such operations. The main and most known being atomic force microscopy (commonly called AFM), optical tweezers and magnetic tweezers [9]. However, these methods are generally invasive or destructive for the biological material studied, which limits or prohibits their use in many cases. AFM was initially conceptualized to operate in a vacuum and nanoscale. Although remaining usable in the air it requires heavy adaptations to work in a liquid and the working scale is rarely adapted to cell biology work. In addition, the piezoelectric base tip handling infrastructure is too large to fit into a microfluidic system. On the other hand, optical tweezers seem ideal to be combined with microfluidic systems, the latter being generally made of transparent materials. However, the use of high intensity focused electromagnetic fields tends to locally heat the medium and damage the manipulated object [10]. In the case of work on fragile biological material, this damage can be significant. Finally, magnetic tweezers can not, with rare exceptions [11], be used with biological material without attaching to or in this material magnetic 
field sensitive bodies. It is quite obvious that this makes it a very intrusive technique, which is often harmful for studies in biology.

The wide variety of possible actions with a micro-robot - manipulation and movement of material in $2 \mathrm{D}$ and $3 \mathrm{D}$ by direct mechanical forces application (by pressing or pushing) [12]-[15] or indirect ones (via hydrodynamic flow generation) [16]-[20] - make it a very versatile tool having a wide range of possible applications. The microscopic swimmer moving on a smaller similar scale biological sample, and study material should be able to apply force at scales similar to the stresses naturally supported by this material, and thus avoid any risk of damage. Especially since microrobots need only a weak magnetic field to function - less than ten milliTesla. And it integrates naturally into a microfluidic device, offering a very suitable environment for work in biology.

Our objective is to develop a new non-contact micromanipulation tool towards applications in cells studies. It should allow precise manipulation and characterization of cells as much individual than at group, and the possibility to have automated functions. As first step we've proposed a design for a rotating helical microswimmer capable of generating vortical flow which allows highly stable noncontact manipulation possibilities [21]. But this version lost the ability to move, and manipulate, in 3D. In this paper we propose the improvement applied to this swimmer to recover the 3D mobility, and so allowing non-contact 3D manipulation of particles. As for previous work we use microfluidic chip as controlled and stable workspace for the development of this tool. It will be our in-vitro environment, and will be useful for future use with biological material.

In this paper we will explain first how we have evolved design and fabrication process of the swimmer. Then we will develop characterization of 6 different models of swimmer we have conceptualized. And finally we will show 3D handling and manipulation promising toward future biological applications. 


\section{Materials and methods}

\subsection{Design and modeling}

The major idea of our previous works [21] is to inverse the central part of the helical micro-swimmer. We expected that this inverted part made local counter-flux opposed to the global hydrodynamics around the robot. This generates local vortices near the robot which enable to trap particle. As the first tests show that the vortex effect is too small compared to the propulsion power of these models. We chose to add a functional head at each side of the models, helping stabilization of side trap and allowing head trap. The results of these first experiments confirmed the possibilities of the concept. However the demonstrated mobility and non-contact manipulation performances are promising but still limited to 2D manipulation. In this section, we show how to overcome this limitation and extended the concept to 3D manipulations.

Table 1. Design evolution

\begin{tabular}{|l|c|c|c|}
\hline & Simple helix [13] & RT3 [21] & RT4 (this work) \\
\hline Fabrication direction & horizontal & horizontal & vertical \\
\hline Fabrication support & no & yes & yes \\
\hline Coating & Cr/Ni ebeam & Cr/Ni ebeam & Cr/Ni sputtering \\
\hline 3D motion & yes & no & yes \\
\hline 3D manipulation & no & no & yes \\
\hline Maximum force range & $\sim 50 \mathrm{pN}$ & not know & $>100 \mathrm{pN}$ \\
\hline
\end{tabular}

\subsubsection{Refined model}

The proposed design in this work was aimed to enable 3D propulsion and manipulation while keeping the major functionalities for the 2D non-contact manipulation which are multimodal (including both the head and side entrapment), stable and non-contact manipulation shown in the previous work [21]. In addition, we kept the bi-helix design meant for the double helical geometry inspired by DNA's double helix. The bi-helix contributes to improve the propulsion stability and smooth response of the swimmer to the magnetic field by reduced wobbling effects due to the 
deviation of magnetization axis to the axis of rotation especially at low propulsion frequency regime.

More details could be found on our previous work [21].
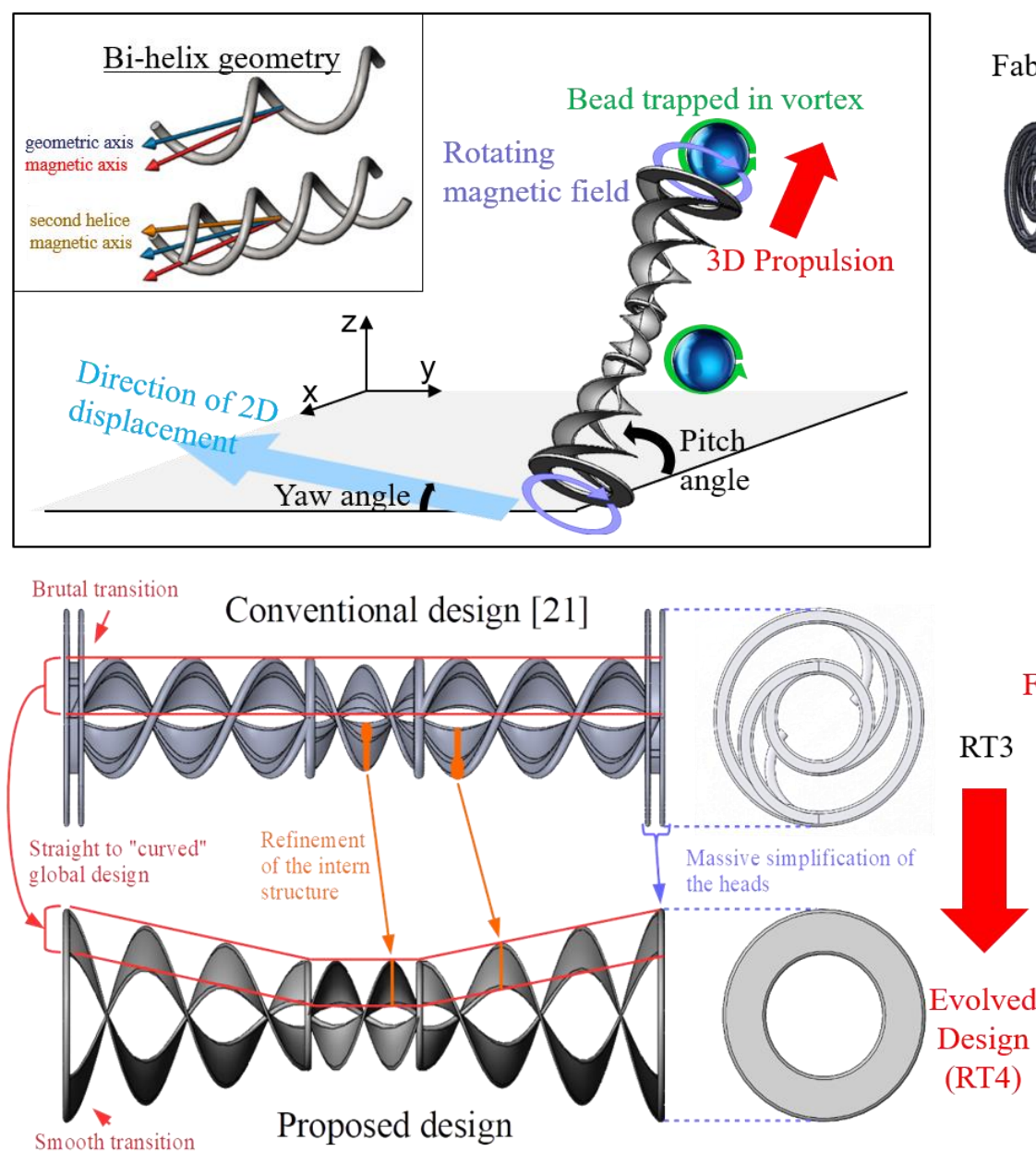

Fabrication in horizontal plane

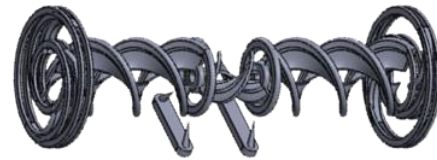

RT3

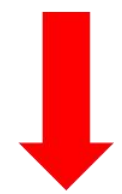

Evolved

Fabrication

(RT4)

Fabrication in vertical axis

Figure 1: Schematic concept of the proposed system with the evolved design and fabrication from RT3 to RT4. The proposed concept is to enable 3D propulsion while keeping multimodal noncontact manipulation capabilities. The overall dimensions of the RT4 model take the dimensions of the "D" variants of the RT3 model [21]. Otherwise, we made three main changes in the design. First of all a global refinement of the structure to lighten the swimmer. Secondly we do not find on the RT3 that complex design for the head have a real utility, so we reduce it to its simplest form : a ring. Finally we found that breaks in hydrodynamic generated by the heads is too strong in RT3 due to brutal transition so we design the external propeller to make a slope between the head and the central propeller.

Figure 1 shows the design modification to recover 3D propulsion and manipulation. Starting from the RT3 variant which had the best results we took the general geometry ratings (number of helix, number and length of pitch) for the realization of the next model, named RT4. In order to recover the 
3D displacement, we modified some geometrical parameters by relying on several hypotheses: The first, reasonable one, is to think that the simplest approach to improve the propulsion / weight ratio remains to refine the swimmer, and therefore reduce its weight. In that case the lower limit of resolution is the voxel size of 3D nanolithography by two-photon absorption (Nanoscribe, $\mathrm{GmbH}$ ).

Also, the 3D model has been reduced to a minimum, and the bulk of the swimmer's volume comes from the voxel via the product of convolution of the latter with the 3D model. This results in propellers with a thickness of $0.2 \mu \mathrm{m}$. In addition, Since the utility of a complex head as for the RT3 model has not really been proven, we have decided to make a simple ring with the thickness of $0.5 \mu \mathrm{m}$, outer diameter $16 \mu \mathrm{m}$ and width $3 \mu \mathrm{m}$. A second hypothesis assumes that the brutal cuts in the RT3 model, especially at the head, can only perturb the generated vortical flow, and thus weaken the net propulsion. We therefore smoothed the swimmer's geometry so that the outer propellers made a slope between the head and the central propeller, as shown in Figure 2.
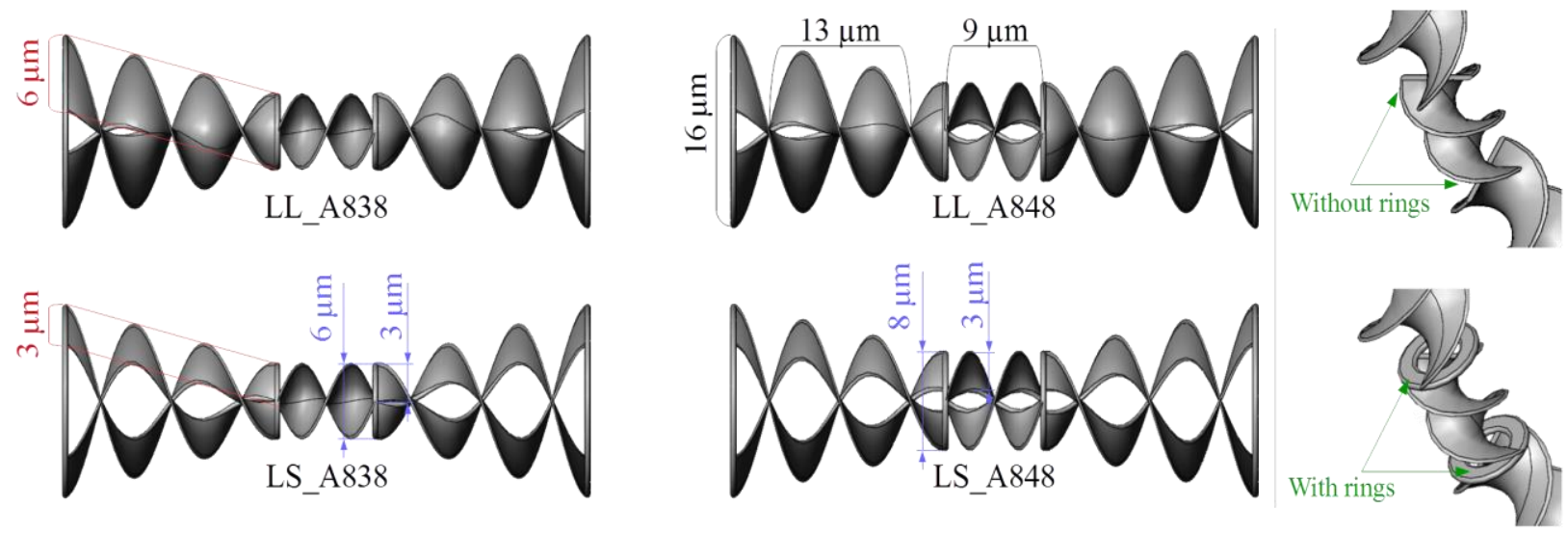

Figure 2: Variants on the RT4 model. The overall dimensions of the RT4 model take the dimensions of the "D" variants of the RT3 model and are shown with black ribs. Variants with "LL" nomenclature are variants with wide propellers where the variants with "LS" nomenclature are those with fine propellers. This distinction is illustrated with red ribs. The next three digits represent the outer radius in micrometer of each piece of the helix, so "838" means that the helix has a radius of 8 $\mu \mathrm{m}$ at the heads and $3 \mu \mathrm{m}$ at the central helix . Likewise " 848 " implies that the inverted central portion has a radius of $4 \mu \mathrm{m}$ while keeping the lateral ends at $8 \mu \mathrm{m}$. Finally the "A" implies the presence of a reinforcement ring at the junction between the propellers, as shown in green on the right. There are two variants not illustrated here, the LS_838 and LL_838, which are exactly the same as, respectively, the variants LS_A838 and LL_A838 with the exception of the absence of the reinforcement ring. 
However we have comparison as main source of discrimination, which requires creating several variants around the model. A first variation allows us to study the effect of the width of the propellers, the parameter for the moment neglected. We have therefore separated the variants into two categories: a category LS having helices of a width equivalent to the ring forming the head, i.e. $3 \mu \mathrm{m}$, and another LL having double-width helices, namely $6 \mu \mathrm{m}$. A second comes from a concern about the strength of the attachment between the lateral and central propellers. We are interested here in the difference between a variant "naked" and another having a reinforcement ring (variant "A"). A third is offered by the presence of the reinforcement ring, which allows us to enlarge the outer diameter of the central helix from $6 \mu \mathrm{m}$ (variant "838") to $8 \mu \mathrm{m}$ (variant "848"). These variations are shown in Figure 2.

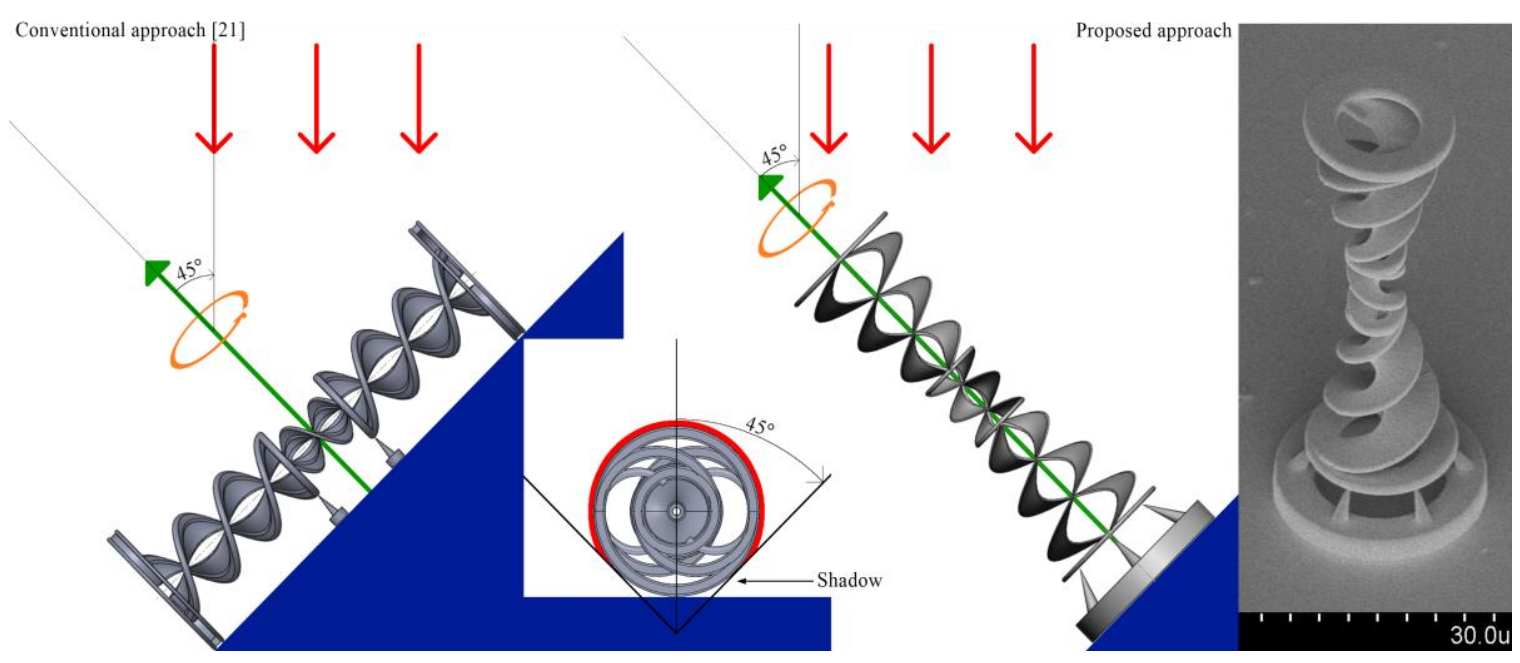

Figure 3: Schematics of the shading problem during metallic deposits and SEM image. On the left, the method used for the RT3 model. The resin core being printed "lying down" on the substrate (in indigo), the rotation (in orange) of this support according to the normal to its surface (in green) is not sufficient to suppress the shadowing effect during the deposition metallic (in red). In the middle, a view of the spirit of the swimmer's area covered by the metal deposit is indicated in red. In the middle right, according to the same color code as on the left, the situation with the model RT4 printed "standing". On the right, the SEM image of the LL_A838 variant printed vertically and metallized by sputtering.

\subsubsection{Vertically standing structures}

A consequent defect requiring a lot of anticipations for mixed results coming from the 3D lithography process. The crosslinking of the resist is made on a non-spherical voxel in the form of a 
prolate spheroid of $1.0 \mu \mathrm{m}$ over $0.4 \mu \mathrm{m}$. The volume of this voxel being considered as an infinitesimal point by the printing software, it generates strong deformations when the details of the model are near the micrometer as the final morphology is the product of convolution between the $3 \mathrm{D}$ model and this voxel. In the framework of the RT3 model, we anticipated this distortion on the 3D model. This deformation is essentially harmful in the context of an impression of the swimmer "lying down" because it is not uniform by rotation around the geometrical axis of the helix. So we decided to pick up the swimmer and print it vertically. This decision required the modeling of a new medium but allowed two major advantages. The first is a considerable reduction of the harmful effect of the deformation since it becomes homogeneous in rotation. This allows us to no longer have to torture the $3 \mathrm{D}$ model before the printing phase and ensure better reproducibility of this model in the resin. The second, which is related to the next point, is that this configuration no longer masks a swimmer's side to coating processes.

\subsubsection{Uniform coating}

Beyond the deformation of the swimmer's resist core, it is important to consider the metallization processes, essentially for the ferromagnetic layer. The previous models saw their ferromagnetic layer made by physical vapor deposition by electron beam evaporation (EBPVD). This technique deposits the material at a fixed angle, which, in the case of our swimmers, makes the ferromagnetic layer grow on one side of the resist core. This results in offsets between the centroid of the total volume and the volume of ferromagnetic and the center of inertia of the swimmer, and therefore instabilities. In an attempt to reduce this effect, the deposition was made with a rotating angle, however the swimmer's extended position produces shadowing phenomena that are difficult to compensate, as shown in Figure 3. The rise of the swimmer detailed previously allowed us to eliminate this shadowing and allow, always with a rotating angle, to achieve a much more homogeneous layer and especially centered on the same geometrical axis as the resin core. 


\subsection{Fabrication process}

The swimmers were modeled with a CAD design software (SolidWorks $\subset$ ), then exported to the proprietary format of the 3D nanolithography tool (Photonic professional GT, Nanoscribe GmbH). The core of the robot is made of epoxy-based polymer resist (IPG 780) by two-photon 3D laser lithography on a glass substrate. To ensure uniform metallization, we decided to have metallization done by cathodic sputtering of two metal layers (Cr/Ni 20/200 nm). This physical vapor deposition technique allows a much more uniform recovery than the EBPVD used previously [22]. Fully exploiting the new vertical geometry of manufacture, this technique theoretically allows us to have a greater homogeneity of the metal layer than for the previous models. Finally, to reinforce the effect of the magnetic field we use the hysteresis of the nickel to impose a permanent magnetization on the swimmer. For this, we applied to the swimmer a magnetic field of $200 \mathrm{mT}$ aligned with the geometric axis of the helix.

Our microfluidic environment is reduced to its simplest form, a unique isolated chamber of a cylinder of $1 \mathrm{~mm}$ height and $6 \mathrm{~mm}$ diameter filled with isopropanol. It is made of a unique layer of PDMS bound on a glass substrate. To allow fluidic filling we made a single little hole of $\sim 100 \mu \mathrm{m}$ diameter on the side of the chip. Then we integrate manually the swimmer before the plasma bonding of the PDMS on the glass.

\section{Characterizations}

As this work is an improvement of our previous one, we will focus on propulsion force as a limiting factor for $3 \mathrm{D}$ propulsion and manipulation. In this section, we will show the propulsion characterizations of the microswimmers to reveal the generated propulsion force. And, as we only have indirect information from our experimentation, we have to recover force from gravity compensation, so we have to discuss a mass estimation of our swimmers. 


\subsection{Mass estimation}

Table 2. Theoretical volume and mass of swimmers

\begin{tabular}{|l|c|c|c|c|}
\hline & $\mathrm{V}_{\text {model }}\left[\mu \mathrm{m}^{3}\right]$ & $\mathrm{S}_{\text {model }}\left[\mu \mathrm{m}^{2}\right]$ & $\mathrm{V}_{\text {total }}\left[\mu \mathrm{m}^{3}\right]$ & $\mathrm{M}_{\text {totale }}[\mathrm{ng}]$ \\
\hline LS_838 & 298 & 2034 & 949 & 2.88 \\
\hline LS_A838 & 318 & 2123 & 997 & 3.01 \\
\hline LS_A848 & 329 & 2351 & 1081 & 3.31 \\
\hline LL_838 & 374 & 2444 & 1156 & 3.48 \\
\hline LL_A838 & 395 & 2534 & 1206 & 3.61 \\
\hline LL_A848 & 422 & 2859 & 1337 & 4.05 \\
\hline
\end{tabular}

With the data of volume and surface extracted from 3D modeling and the knowledge of swimmers manufacturing processes it becomes possible to calculate a theoretical value of the volume and the mass (Table 2) of the swimmers. Despite this we have made some simplifications because some data are inaccessible :

- The volume of resin partially takes into account the deformation due to the printing voxel according to the formula: $V_{\text {res }}=V_{\text {mod }}+S_{\text {mod }} \times$ voxel error. We have chosen to consider a uniform voxel error of $0.4 \mu \mathrm{m}$ although the voxel is oblong because the model essentially consists of a standard surface close to the $\mathrm{z}$ axis, the error along this axis has the greatest impact. We are conscious of maximizing the error here, which generates a positive error on the calculated final mass.

- Chromium and nickel volumes were calculated using the same formula: $V_{m e t}=S_{m o d} \times t_{\text {thickness }}$ of metal. We are aware here of neglecting the surface increase due to the deformation of the voxel, and in the case of nickel that due to the deposition of chromium, which generates a negative error.

- The resin mass was calculated by producing the product of the resist volume and the density of the crosslinked resist as determined experimentally by Antoine Barbot [13] : $\rho_{\text {resist }}=1.1028 \mathrm{~kg} \cdot \mathrm{dm}^{-3}$.

- Metal masses were calculated by considering their bulk density values. 


\subsection{D Propulsion}

The propulsion of the swimmer is done by a rotating magnetic field generated with Helmholtz coils assembly. Our setup generates a magnetic field of $6 \mathrm{mT}$. The generated torque on a magnetized body in a magnetic field is given by :

$$
\tau_{m}=\mu_{0} V \vec{M} \times \vec{H}
$$

With $\mu_{0}$ the permeability of free space, the $V$ volume of the body (in our case the volume of the ferromagnetic Ni coating), $\vec{M}$ the magnetization of the body and $\vec{H}$ the applied magnetic field. By rotating the field, we made the swimmer rotating along its helical axis, generating a propulsion flux. This propulsion was studied by Edward M. Purcell [23] and follow the matrix :

$$
\left(\begin{array}{l}
F \\
\tau
\end{array}\right)=\left(\begin{array}{ll}
A & B \\
B & C
\end{array}\right)\left(\begin{array}{c}
v \\
\omega
\end{array}\right)
$$

With $F$ the sum of external forces applied to the swimmer, $\tau$ the couple applied to the swimmer, $v$ the velocity of the swimmer along his helical axis, $\omega$ the rotation speed of the swimmer and A, B and $\mathrm{C}$ configuration-dependent constants. Here the external forces are the resultant gravity forces $P_{v}(\theta)$ (weight and buoyancy) applied to the direction of the velocity vector (with the angle between the weight vector and the velocity vector) and a drag force $T=T_{0} v$ of its displacement in the fluid. And the torque $\tau$ is the torque $\tau_{m}$ generated by the rotating magnetic field. This changes the equation 2 to:

$$
\begin{aligned}
P_{v}(\theta)+T_{0} v & =A v+B \omega \\
\tau_{m} & =B v+D \omega
\end{aligned}
$$




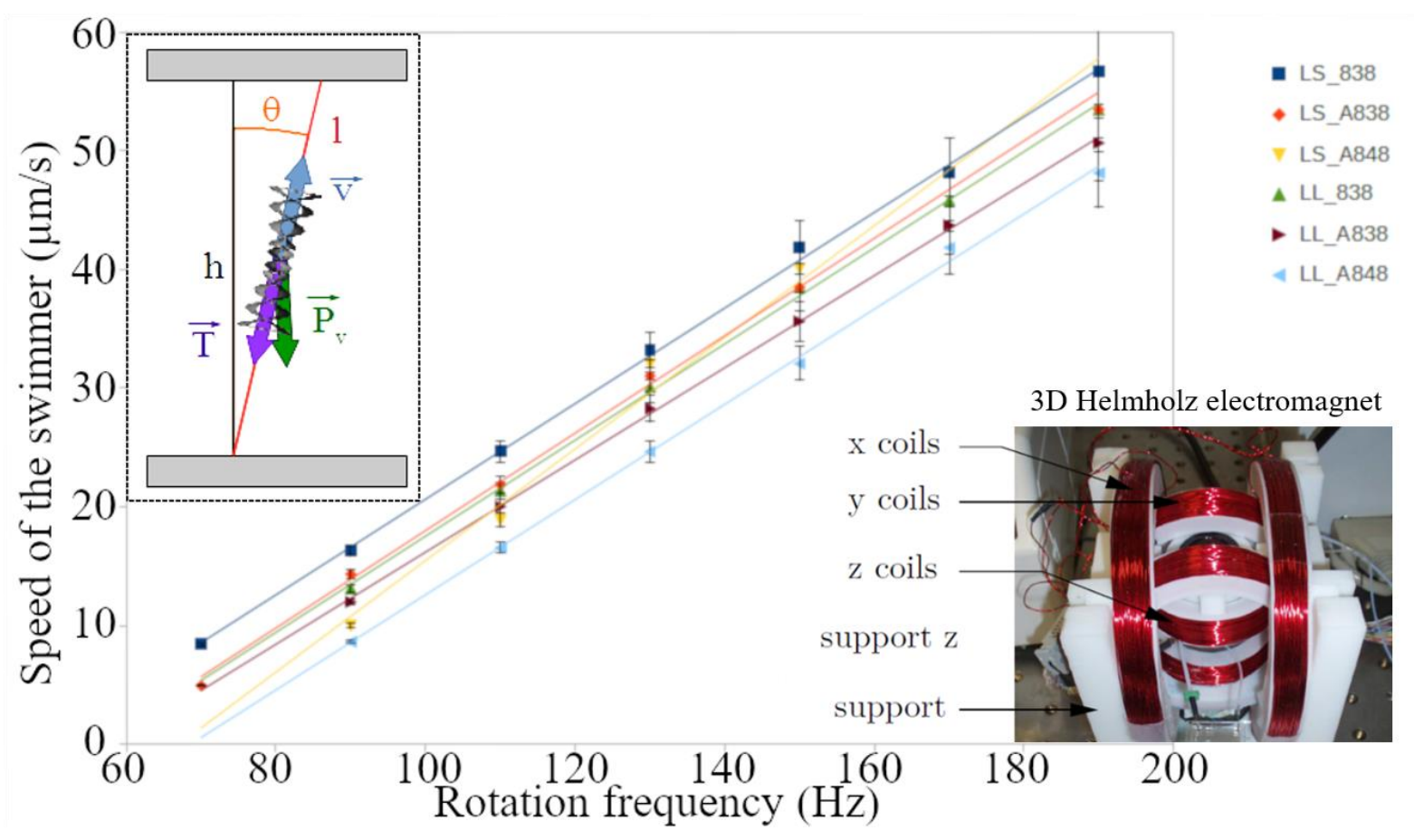

Figure 4: Swimmer's 3D characterization. On the inset figure left, a schematic of the manipulation with $h$ the height of the characterization chamber, $\theta$ the angle between the normal to the surface and the speed direction, $l$ the achieved trajectory, $\nu$ the speed of the swimmer, $P_{v}$ the resultant gravity force and $T$ the drag force. The measurement time sped to travelled distance $l$ at a given frequency gives the speed $v$ of the swimmer. On the right, the speeds according to the imposed rotational frequency for the six variants of RT4. We can observe that we are seeing a linear form as expected with the equation 5. However the results of variant LS_A848 differ greatly from others and are possibly a measuring artifact. The inset right image shows the 3D Helmholtz electromagnetic coils to generate $3 \mathrm{D}$ rotating magnetic field $[13,21,24]$.

If we are in the situation of the couple $\tau_{m}$ is strong enough to impose its rotation speed to the swimmer, we can consider that $\omega$ is a constraint, leave beside the equation 4 and reformulate the equation 3 :

$$
\begin{array}{rlrl}
v & =\frac{-B \omega+P_{v}(\theta)}{A-T_{0}} & C_{1} & =\frac{-B}{A-T_{0}} \\
\Leftrightarrow v=C_{1} \omega+C_{2}(\theta) & C_{2}(\theta) & =\frac{P_{v}(\theta)}{A-T_{0}}
\end{array}
$$

Thus we have a linear relation between the swimmer's speed and its rotation frequency as long as we stay in a frequency range where the torque generated by the magnetic field on the swimmer is sufficient to force a rotation frequency equal to that of the magnetic field. In order to determine the parameters $C_{1}$ and $C_{2}(\theta)$ we measured the time that the swimmer has to travel the height of the 
characterization chamber which is $1 \mathrm{~mm}$ with an angle $\theta=12^{\circ}$ at different frequencies of rotation. As the measurement was chronometered with the eye, it was necessary to have an angle $\theta>0$ so that when the swimmer comes into contact with the upper surface of the chip, he changes his mode of movement to the surface motion (spintop) [24]. This displacement from the mode shift is very visible thus easy to chronometer the travel time. It remains reasonable to consider a measurement error of the order of one second. From these times and a calculation of the travelled distance we can go back to a value of speed dependent on the frequency. These experimental results are summarized in figure 4. A linear regression of these results allows us to find the parameters and presented in Table 3 with a very high degree of certainty, as evidenced by the value of the coefficient of determination $\mathrm{R}^{2}$. Moreover, the knowledge of the mass of the swimmers, studied previously, allows us also to extract the value of the constants $B$ and $A-T_{o}$. These last two constants are essential because they allow us to free ourselves from the dependency of characterization. It becomes possible to calculate the swimmer's 3D swim evolution from these constants and its mass, an essential step for automating 3D mobility.

Table 3. Constants of equation 5 for an angle of inclination of $12^{\circ}$

\begin{tabular}{|l|c|c|c|c|c|c|}
\hline & LS_838 & LS_A838 & LS_A848 & LL_838 & LL_A838 & LL_A848 \\
\hline$C_{1}[\mathrm{~nm}]$ & 418 & 418 & 517 & 423 & 395 & 391 \\
\hline$C_{2}\left(\theta=12^{\circ}\right)\left[\mu \mathrm{m} \cdot \mathrm{s}^{-1}\right]$ & -21.1 & -23.8 & -36.7 & -24.9 & -23.5 & -26.4 \\
\hline $\mathrm{R}^{2}$ & 0.9997 & 0.9985 & 0.9912 & 0.9999 & 0.9994 & 0.9997 \\
\hline$P_{\mathrm{v}}\left(\theta=12^{\circ}\right)[\mathrm{pN}]$ & 21.7 & 23.2 & 25.6 & 26.7 & 27.7 & 31.2 \\
\hline$A-T_{0}\left[\mathrm{~g} \cdot \mathrm{s}^{-1}\right]$ & 1.03 & 0.975 & 0.698 & 1.07 & 1.18 & 1.18 \\
\hline$B\left[\mathrm{ng} \cdot \mathrm{m} \cdot \mathrm{s}^{-1}\right]$ & 431 & 408 & 361 & 453 & 466 & 461 \\
\hline
\end{tabular}

In order to have a basis of comparison in terms of the force generated by the swimmer in rotation we will consider the equation 5 considering the limit case where the swimmer is at equilibrium and therefore at a speed of zero, we obtain the relationship:

$$
\begin{aligned}
C_{1} \omega & =-C_{2}(\theta) \\
\Leftrightarrow \frac{B}{A-T_{0}} \omega & =\frac{P_{v}(\theta)}{A-T_{0}} \\
\Leftrightarrow \quad B \omega & =P_{v}(\theta)
\end{aligned}
$$



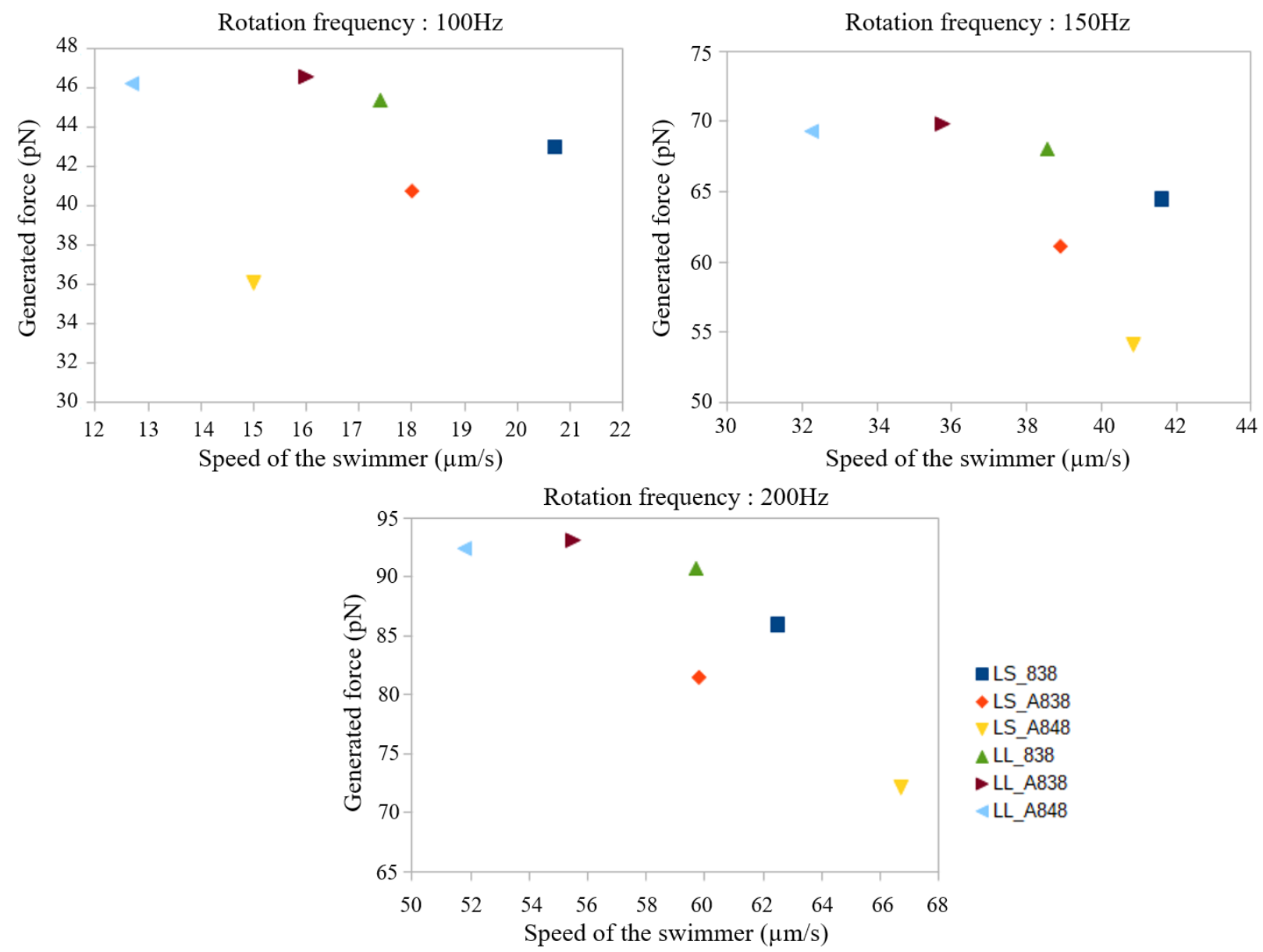

Figure 5: Comparative graphs of the RT4 variants according to the Force vs. Speed at given rotation frequencies and with an inclination of $12^{\circ}$ relative to the weight vector. The force is calculated according to the formula $F=B \omega$ and the speed according to the equation 5 with parameters values of the table 3. There is a clear dominance of the variants LS_838, LL_838 and LL_A838. Once again results for the variant LS_A848 do not have the same evolution as the others which tends to confirm the hypothesis of measurement artifact.

And since we are in the case where the swimmer is in equilibrium, we can consider that in this case $P_{v}(\theta)$ is the force generated by the swimmer. Obviously, experimental results show that the swimmer is not at equilibrium at theses frequencies, which means that is more a minimal propulsion force. But 
we can therefore reasonably extrapolate $F=B \omega$ as a force value generated by the swimmer for comparison between variants. The graph in Figure 5 compares the variants according to the swimmer's force and speed torque running at frequencies of 100,150 and $200 \mathrm{~Hz}$. We confirm that the Figure 5 characterizations were all below the step-out frequencies of the microswimmers. The Figure 4 shows that swimmers have linear reaction to the frequency on the $70-190 \mathrm{~Hz}$ range while their step-out frequencies are around $250-300 \mathrm{~Hz}$ for these models. These first results seem to disqualify the variants LS_A838 and LL_A848 which have the worst results. The evolution with the frequency of the results of model LS_A848 seems to go in the direction of the hypothesis of an artifact.

The last three models (LL_A838, LL_838 and LS_838) become difficult to discriminate and offer three options for optimization: the LS_ 838 will be preferred on applications requiring speed, the LL_A838 on those requiring power and the LL_838 in those requiring a balance between the two.

\subsection{Simple material manipulation}

As for the previous model, we carried out manipulation experiments in isopropanol with $10 \mu \mathrm{m}$ polystyrene beads. As it is difficult to automate the 3D manipulation, the results were all obtained by manual manipulation of the swimmer.

\subsubsection{D manipulation}

The RT4 model seeking to keep the previous model's 2D features, we expected to have the same handling options. The performed tests have made it possible to qualitatively reproduce the three manipulation options detailed in our previous work [21]. However, if head capture and push / pull options are substantially the same, lateral capture has become more complex. This difference was predictable since we remain on a paradigm of opposition between propulsion flow and counter-flow of capture and that this model sought to improve the propulsion to have a displacement $3 \mathrm{D}$. It has 
therefore become necessary to increase the rotational frequency of the swimmer to effectively capture the same category of beads.

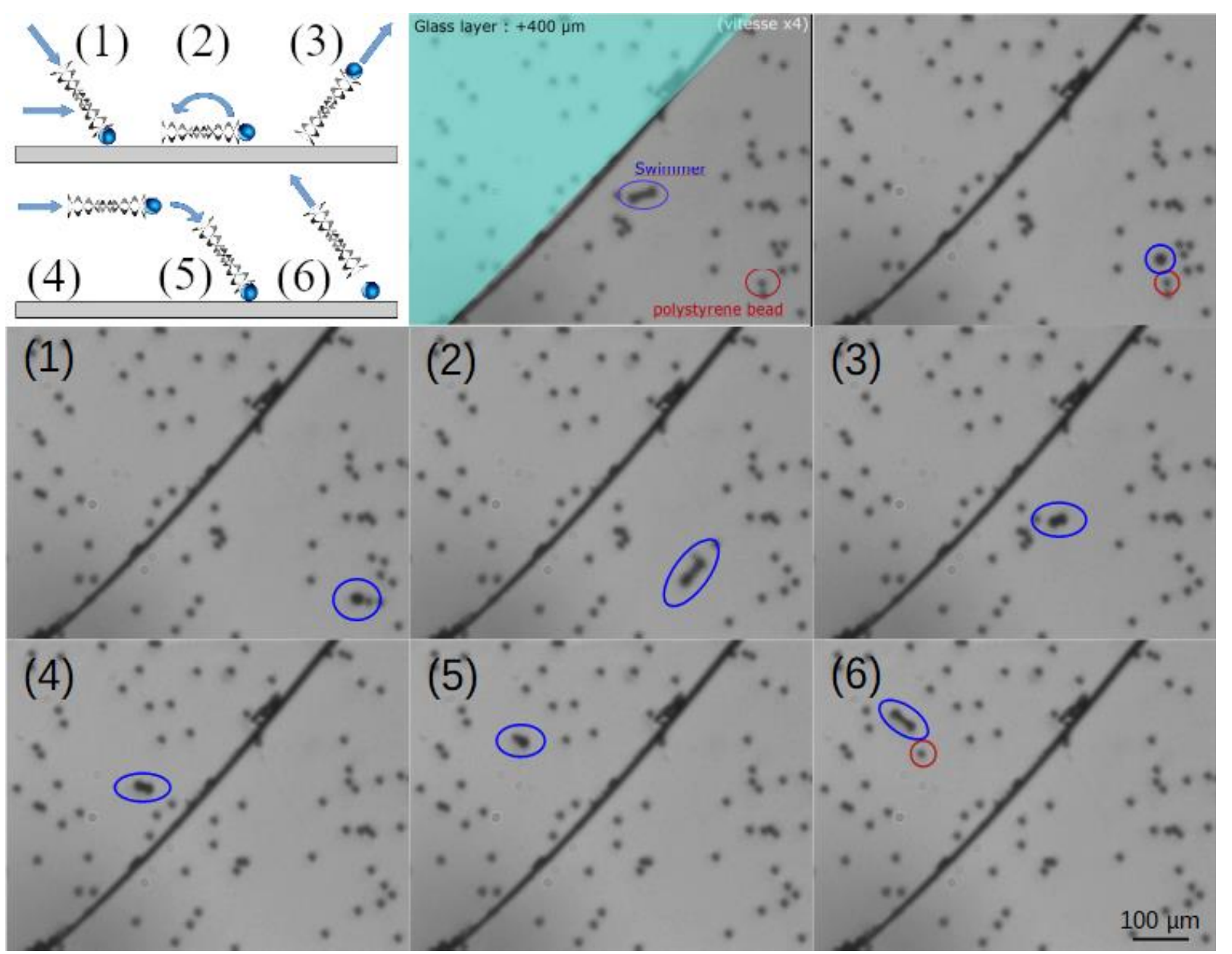

Figure 6: Diagram and screenshots of the steps of capture and positioning of a particle in 3D.

The blue arrows represent the movement of the swimmer. The blue rings highlight the swimmer and the red rings highlight the trapped particle. The black line on the screenshots is the border of a glass plate of $400 \mu \mathrm{m}$ tickness. (1) Capturing the particle, this can be done in "Spintop" or "Swim" mode depending on the situation. It was done in "Spintop" on the screenshot. (2) Without changing the direction of rotation of the swimmer it is turned $180^{\circ}$ on its Pitch angle to switch to "Swim" mode. (3) The swimmer takes off in "Swim" mode with the captured particle at his head. (4) The swimmer is controlled in "Swim" mode, with all the maneuverability offered by this mode of displacement. (5) The swimmer goes to the place where the particle must be released. (6) Once the particle is positioned, the direction of rotation of the swimmer is reversed so that it takes off in the other direction leaving the particle in place.

\subsubsection{D manipulation}

The RT3 model had three capture zones, one at the counter-helix and one at each end. We could see in the context of the 2D movement that these capture areas still exist but for the passage in 3D it gets complicated. As explained above, the lateral capture zone is in the state the result of a balance 
between general flow, counter flow and effect of weight. This works in "Spintop", but reversing the direction of rotation of the swimmer, and thus the direction of flow and counter-flow, to enter "Swim" mode breaks this balance and prohibits any stable capture at this point. Likewise, the area at the rear head - i.e. on the side of the propulsion - can no longer be a stable trapping area since the propulsion of the swimmer will expel the particle. There remains only one usable area in 3D: the head zone is the opposite of the propulsion. It becomes complicated to use this swimmer and the maneuver to be done to capture a particle, shown schematically in Figure 6, can be tedious. However, once the particle is captured and the swimmer in the "Swim" situation, this capture is very stable and allows a totally free $3 \mathrm{D}$ manipulation, and a very precise positioning.

In the Figure 6, we can assure that the microswimmer traps a micro bead, moves over the additionally put glass substrate (the height is 400 microns) on the left part (colored part) of the screenshots and successfully placed the bead on top of the glass substrate 400-micron higher than the base substrate on the right side of the screenshots.

\section{Conclusion}

We propose a new helical microswimmer model (RT4) for non-contact 3D manipulation. The previous model (RT3) achieved goal of highly stable hydrodynamics capture by generated vortical flow but lost the ability to move in three dimensions, which de facto limited the manipulation options to two dimensions only. The RT4 has had a refinement of its structure and especially a reflection on its manufacturing protocol. Enhancing both the propulsion force by new design and the surface homogeneity of metallic layer coating allowed the RT4 to recover mobility in three dimensions, the "Swim" mode. However, the complexity of the balancing between the propulsion and capture flows for the lateral zone does not make it possible to use it in "Swim" mode, but the capture zone at the level of the swimmer's head allows a stable capture, and therefore a manipulation, in three dimensions. We keep the side areas for two-dimensional manipulation, with the "Spintop" mode. In this way, this 
model achieves non-contact manipulation in two and three dimensions by hydrodynamic vortices. We believed that this will be promising to serve as on-chip micromanipulator with force characterizations of biological samples. To apply the proposed microswimmer for live cell manipulation, we need to carefully demonstrate and characterize the propulsion and manipulation performance in biological culture medium. The remaining question for biological cell manipulation could be the effect of the stretching due to the vortex on the cell may be too strong, but it'll need proper experimentations for the verification.

\section{Acknowledgments}

We appreciate Ms. Ayako Mizushima and Prof. Yoshio Mita for their supports to sputtering metallic layers at VDEC, The University of Tokyo during the closed period of C2N cleanroom facility.

\section{Funding}

This work was supported by Centre National de la Recherche Scientifque and Universite ParisSaclay. This work is also partly supported by C'Nano IdF DIM Nano-K and a public grant overseen by the French National Research Agency (ANR) as part of the "Investissements d'Avenir" program (Labex NanoSaclay, reference: ANR10-LABX-0035).

\section{References}

[1] P. M. Lintilhac and T. B. Vesecky, "Stress-induced alignment of division plane in plant tissues grown in vitro," Nature, vol. 307, no. 5949, pp. 363-364, Jan. 1984. 
[2] B.-S. Kim, J. Nikolovski, J. Bonadio, and D. J. Mooney, "Cyclic mechanical strain regulates the development of engineered smooth muscle tissue," Nat. Biotechnol., vol. 17, no. 10, pp. 979-983, Oct. 1999.

[3] C. J. Meyer, F. J. Alenghat, P. Rim, J. H.-J. Fong, B. Fabry, and D. E. Ingber, "Mechanical control of cyclic AMP signalling and gene transcription through integrins," Nat. Cell Biol., vol. 2, no. 9, pp. 666-668, Sep. 2000.

[4] G. Helmlinger, P. A. Netti, H. C. Lichtenbeld, R. J. Melder, and R. K. Jain, "Solid stress inhibits the growth of multicellular tumor spheroids," Nat. Biotechnol., vol. 15, no. 8, pp. 778-783, Aug. 1997.

[5] V. Swaminathan, K. Mythreye, E. T. O’Brien, A. Berchuck, G. C. Blobe, and R. Superfine, "Mechanical Stiffness Grades Metastatic Potential in Patient Tumor Cells and in Cancer Cell Lines," Cancer Res., vol. 71, no. 15, pp. 5075-5080, Aug. 2011.

[6] T. Itabashi, Y. Terada, K. Kuwana, T. Kan, I. Shimoyama, and S. Ishiwata, "Mechanical impulses can control metaphase progression in a mammalian cell," Proc. Natl. Acad. Sci., vol. 109, no. 19, pp. 7320-7325, May 2012.

[7] T. Itabashi, J. Takagi, K. Suzuki, and S. Ishiwata, "Responses of chromosome segregation machinery to mechanical perturbations," Biophysics (Oxf)., vol. 9, no. 0, pp. 73-78, 2013.

[8] K. A. Knouse, K. E. Lopez, M. Bachofner, and A. Amon, "Chromosome Segregation Fidelity in Epithelia Requires Tissue Architecture," Cell, vol. 175, no. 1, pp. 200-211.e13, Sep. 2018.

[9] K. C. Neuman and A. Nagy, "Single-molecule force spectroscopy: optical tweezers, magnetic tweezers and atomic force microscopy,” Nat. Methods, vol. 5, no. 6, pp. 491-505, Jun. 2008.

[10] K. C. Neuman, E. H. Chadd, G. F. Liou, K. Bergman, and S. M. Block, "Characterization of Photodamage to Escherichia coli in Optical Traps,” Biophys. J., vol. 77, no. 5, pp. 2856-2863, Nov. 1999.

[11] R. Blakemore, "Magnetotactic bacteria," Science, vol. 190, no. 4212, pp. 377-379, Oct. 1975. 
[12] T. Kawahara et al., "On-chip microrobot for investigating the response of aquatic microorganisms to mechanical stimulation," Lab Chip, vol. 13, no. 6, p. 1070, 2013.

[13] A. Barbot, "On-Chip Untethered helical Microrobot for Force Sensing Applications," Ph.D thesis, 2016.

[14] E. B. Steager, M. Selman Sakar, C. Magee, M. Kennedy, A. Cowley, and V. Kumar, “Automated biomanipulation of single cells using magnetic microrobots,” Int. J. Rob. Res., vol. 32, no. 3, pp. 346359, Mar. 2013.

[15] J. Zhang, O. Onaizah, K. Middleton, L. You, and E. Diller, "Reliable Grasping of ThreeDimensional Untethered Mobile Magnetic Microgripper for Autonomous Pick-and-Place," IEEE Robot. Autom. Lett., vol. 2, no. 2, pp. 835-840, Apr. 2017.

[16] S. Floyd, C. Pawashe, and M. Sitti, "Two-Dimensional Contact and Noncontact Micromanipulation in Liquid Using an Untethered Mobile Magnetic Microrobot," IEEE Trans. Robot., vol. 25, no. 6, pp. 1332-1342, Dec. 2009.

[17] T. Petit, L. Zhang, K. E. Peyer, B. E. Kratochvil, and B. J. Nelson, "Selective Trapping and Manipulation of Microscale Objects Using Mobile Microvortices," Nano Lett., vol. 12, no. 1, pp. 156-160, Jan. 2012.

[18] H.-W. Tung, K. E. Peyer, D. F. Sargent, and B. J. Nelson, "Noncontact manipulation using a transversely magnetized rolling robot,” Appl. Phys. Lett., vol. 103, no. 11, p. 114101, Sep. 2013.

[19] Z. Ye and M. Sitti, "Dynamic trapping and two-dimensional transport of swimming microorganisms using a rotating magnetic microrobot," Lab Chip, vol. 14, no. 13, pp. 2177-2182, 2014.

[20] H.-W. Tung, R. Pieters, D. F. Sargent, and B. J. Nelson, "Non-contact manipulation for automated protein crystal harvesting using a rolling microrobot," in 2014 IEEE International Conference on Robotics and Automation (ICRA), 2014, vol. 19, no. 3, pp. 2092-2092. 
[21] A. Paris, D. Decanini, and G. Hwang, “On-chip multimodal vortex trap micro-manipulator with multistage bi-helical micro-swimmer," Sensors Actuators A Phys., vol. 276, pp. 118-124, Jun. 2018. [22] D. M. Mattox, "Physical vapor deposition (PVD) processes," Met. Finish., vol. 98, no. 1, pp. 410-423, Jan. 2000.

[23] E. M. Purcell, "The efficiency of propulsion by a rotating flagellum,” Proc. Natl. Acad. Sci., vol. 94, no. 21, pp. 11307-11311, Oct. 1997.

[24] A. Barbot, D. Decanini, and G. Hwang, “On-chip Microfluidic Multimodal Swimmer toward 3D Navigation,” Sci. Rep., vol. 6, no. 1, p. 19041, May 2016. 